\title{
Identification of Nigerian English idioms: a methodological perspective
}

\begin{abstract}
Sociolinguists who study varieties of language often need to make decisions about whether a particular linguistic element or expression constitutes a feature of the variety being studied. Comparing the specific variety, for instance, a variety of English, with the parent or native form of English is generally carried out to examine for systematic differences between the two forms. The task becomes more challenging when the feature of interest involves figurative meaning such as idioms and idiomatic expressions. In a recently completed project that sought to examine idioms in Nigerian English, questions and issues about methodology were raised. Among these were the questions of how idioms in the regional variety could be identified as distinct from idioms that are originally found in the parent variety, and more importantly, what evidence could be offered to support claims that a particular idiom is a feature of the regional variety. In this article, we discuss the issues connected with the difficulty of examining idioms in a specific variety of English known as Nigerian English, and propose a framework for evaluating the idioms.
\end{abstract}

Keyword: Nigerian English idioms; Methodological perspective; Sociolinguists 